used to identify the number of non-elective hospital admissions, reason and length of admission.

Results In JOPCC there were 45 non-elective hospital admissions compared to 70 admissions in SOC. The main reason for admission in both groups was for new complications of diagnosis: JOPCC $84.4 \%$; SOC $85.5 \%$. Complications of treatment were more common in the SOC patients; JOPCC $10.5 \%$; SOC $26.7 \%$. The average length of admission was significantly shorter $(\mathrm{p}=0.0015)$ in the JOPCC (4.8 days).

Conclusions JOPCC appear to reduce both the number and length of non-elective admissions. This may be partly attributed to fewer patients actually receiving treatment or having previous access to palliative care teams; resulting in fewer admissions. Further research comparing non-elective admissions within 12 months from first consultation with a larger sample size, confining data to a single site-specific cancer diagnosis will allow further conclusions to be made.

\section{P-227 COMBINED ONCOLOGY AND PALLIATIVE CARE CLINICS; PATIENT PLACE OF DEATH}

1,2Emily Russell, 1,2 Lucy Adami, 1,2Duncan Paterson, 1,2,3 Jane Gibbins, ${ }^{2,3}$ Richard Ellis, ${ }^{1,2,3}$ Carolyn Campbell, ${ }^{1,2,3}$ Rachel Newman, ${ }^{1,2,3}$ Deborah Stevens. ${ }^{1}$ Cornwall Hospice Care, St Austell, UK; ${ }^{2}$ Royal Cornwall Hospitals NHS Trust, Truro, UK; ${ }^{3}$ University of Exeter Medical School, Exeter, UK

\subsection{6/bmjspcare-2019-HUKNC.249}

Background Combined oncology and palliative care clinics (COPCC) have developed in Cornwall to enable early palliative medicine input into the care of cancer patients. The evidence suggests referrals to palliative care are usually late (Bennett, Ziegler, Allsop, Daniel et al., 2016), despite growing evidence that earlier palliative care reviews enhance patient quality of life and in some cases survival (Temel, Greer, Muzikansky, Gallagher et al., 2010). The majority of patients express a preference to die either in their own home or the hospice (Gomes, Higginson, Calanzani, Cohen et al., 2012; Gao, Ho, Verne, Glickman et al., 2013; Gomes, Calanzani, Gysels, Hall et al., 2013), yet unfortunately most die in acute hospitals (Cohen, Pivodic, Miccinesi, Onwuteaka-Philipsen et al., 2015). The aim of this review was to determine the place of dying phase of care for patients reviewed in the COPCC.

Method A retrospective database review identified place of death for 675 consecutive patients seen in COPCC between 2012 and 2017.

Results $45 \%$ died at home or nursing home, $26 \%$ died in the hospice and 29\% died in hospital (21\% acute, $8 \%$ community). In comparison nationally, $37 \%$ of patients with cancer died at home or nursing home, $17 \%$ died in palliative care institutions (i.e. hospice) and 44\% died in hospital.

Conclusions COPCC aim to provide holistic palliative care to control symptoms, address information needs, help with psychological distress and guide advance care planning. COPCC allow patients with advanced cancer to be referred earlier to the community palliative care teams or hospice services. Patients who are reviewed in COPCC appear to be more likely to die at home, nursing home or hospice and less likely to die in an acute hospital compared to national data for patients with a cancer diagnosis. COPCC may help patients to die in their preferred place of death. Patients with non-cancer diagnoses such as end stage heart failure may also benefit from combined clinics with palliative care. Further work is needed to explore this.

\section{P-228 LINKING HOSPITAL TO HOME AT END OF LIFE}

Alex Van Der Walt, Rebecca Ford. Oxford University Hospitals Trust, Oxford, Oxfordshire

\subsection{6/bmjspcare-2019-HUKNC.250}

Background No Occupational Therapists (OTs) previously in Hospital Palliative Care Team (HPCT) and lack of continuity for patients and their families from leaving hospital to being at home for end of life care (EOLC).

- It was recognised that OTs were needed as an essential part of the HPCT;

- Needed to improve upon patient and family's experiences of EOLC in the John Radcliffe Hospital.

$\operatorname{Aim}(s)$

- To provide support and continuity of care for patients and families throughout patient's EOLC journey from hospital to home;

- To provide education and support to hospital staff in regards to palliative and EOLC;

- To promote role of OT in palliative care within the trust and nationally.

Methods

- Networking with other OTs working in palliative care and sharing ideas;

- Qualitative data collected - formally from staff using an anonymous form and informally from patients and relatives;

- Planning on initiating a qualitative research project using a research assistant to interview families and loved ones.

\section{Results}

- 237 patients seen in the first year;

- $100 \%$ of staff agreed that input from the palliative care OTs improved the quality of EOLC for palliative patients;

- Excellent informal feedback from patients, relatives and staff across the hospital and in the community.

Conclusions Project demonstrated that OTs in the HPCT have a positive impact on the EOLC received by patients in the trust and into the community. We are one of the only OT teams that follow patients through their entire journey from hospital to home. The findings of our project supports ambition 4 of the Ambitions for Palliative and End of Life Care ensuring care is co-ordinated.

This project will be of interest to palliative care professionals across the country as it is displaying innovative practice and has so far achieved excellent results and feedback.

\section{P-229 DOROTHY HOUSE HOSPICE CARE AND ROYAL UNITED HOSPITAL - COMPASSIONATE COMPANIONS HOSPITAL END OF LIFE CARE}

Sadie Hall, Wendy Meilton. Dorothy House Hospice Care, Bath, UK

\subsection{6/bmjspcare-2019-HUKNC.251}

The Compassionate Companions Service is a new service that provides specially-trained volunteers who offer support, compassionate listening, comfort and companionship to patients in their last days of life at the hospital, who do not want to die alone. They will also provide respite companionship support for patients' families so that loved ones can recuperate during what is a psychologically and physically exhausting time. 